\title{
＜原著論文 (Short communication) $>$ \\ The Fatty Acid Composition of Fish Consumed in Dalian- and Hangzhou-City, China
}

Weiyu Zhang ${ }^{1)}$, Rong Xia ${ }^{2)}$, Tetsuro Nagasawa ${ }^{3)}$, Yueji Sun $^{4)}$, Kei Hamazaki ${ }^{3) *}$, Jun $\mathrm{Wu}^{4)}$ and Tomohito Hamazaki ${ }^{3)}$

${ }^{1)}$ Pharmaceutics Office in Academy of Medicine, Changchun University of Traditional Medicine, Changchun-city, Jilin-province, China

${ }^{2)}$ Basic Medical College of Zhejiang Chinese Medical University, Binjiang Hangzhou 310053, Zhejiang Province, China,

3) Division of Clinical Application, Department of Clinical Sciences, Institute of Natural Medicine, University of Toyama, Sugitani, Toyama-City, Toyama 930-0194, Japan,

4) Department of Psychology, Dalian Medical University, Dalian-city, Liaoning- province 116044, China

*: Correspondence to: Kei Hamazaki, MD, PhD: Department of Public Health, Faculty of Medicine, University of Toyama, 2630 Sugitani, Toyama 930-0194, Japan; Phone: +81 76434 7279; FAX: +81 76434 5023; E-mail: keihama@med.u-toyama.ac.jp

\begin{abstract}
There was essentially no database on the fatty acid composition of fish available in China. Twenty-seven kinds of fish and 20 kinds were purchased in Dalian- and Hangzhou-city, China, respectively, and edible part was analyzed for the fatty acid composition. Three kinds of fish were purchased in both cities, but they contained markedly different amounts of $n-3$ polyunsaturated fatty acids (PUFA). Those kinds of fish in Hangzhou-city had more n-3 PUFA than those in Dalian-city. This was probably because fish were purchased in winter in Hangzhou-city and in summer in Dalian-city. Our data were limited in terms of the area and season of catch, and the number of species. However, our database would serve as the starting point for the fatty acid composition study of Chinese fish.
\end{abstract}

Key words: $n-3$ polyunsaturated fatty acids, fatty acid composition, Chinese fish, nutrition, database 
Received 2009.7.24; Revised received, 2009.10.26; Accepted 2009.11.6

The importance of $n-3$ polyunsaturated fatty acids (PUFA) in lipid nutrition is well-known. However, there were only a few reports about the fatty acid composition of fish consumed in China, and the numbers of fish investigated were very small: one kind of fish in a report ${ }^{1)}$ and two in another ${ }^{2}$. We performed a case-control study of suicide attempt and n-3 PUFA in Dalian-city, China ${ }^{3)}$, and found that the relative risk of suicide attempt in the highest quartile of eicosapentaenoic acid (EPA) concentrations in the phospholipid fraction in red blood cells was only one eighth of that in the lowest. In that study we could not determine how much n-3 PUFA they ate because there were neither food questionnaires nor databases that would calculate n-3 PUFA intake from their diet. The purpose of the present study was to facilitate to compile a fatty acid database of Chinese fish.

In July and December, 2005, 27 and 20 kinds of popular fish were purchased at local fish and super markets in Dalian- and Hangzhou-city, China, respectively. Dalian-city is in the eastern Liaoning Province of Northeast China, facing Shandong to the south and the Yellow Sea to the east and the Bohai Sea to the west and south. Hangzhou-city is located in the Yangtze River Delta and 180 kilometres southwest of Shanghai. Those fish were stored at $-30^{\circ} \mathrm{C}$ and then analyzed. Edible part (three to five different portions including the dorsal and ventral) of each fish (only one fish sample for each species) was minced and mixed thoroughly. One $\mathrm{g}$ of the mixture was analyzed with 17:0 as an internal standard by the saponification method of the Japan Food Research Laboratories ${ }^{4)}$ with slight modification. After transmethylation of fatty acids with HCl-methanol, the fatty acid composition was analyzed by gas chromatography (GC14A Shimadzu Corporation, Kyoto) with a capillary column DB-225 (length, $30 \mathrm{~m}$; internal diameter, $0.25 \mathrm{~mm}$; film $0.25 \mu \mathrm{m}$; J\&W Scientific, Folsom, CA). The intra-assay coefficient of variance of the saponification method was $5.0 \%$ for EPA and $4.6 \%$ for docosahexaenoic acid (DHA). Data are expressed as means.

Tables 1 and 2 show the fatty acid composition of fish purchased in Dalianand Hangzhou-city, respectively. Three kinds of fish, ribbonfish (Trichiurus haumela), tench (Ophiocephalus argus) and small yellow croaker (Pseudosciaena polyactis), were purchased in both Dalian- and Hangzhou-city. The total n-3 PUFA content found in those three fish in Dalian-city were 280,224 and $366 \mathrm{mg} / 100 \mathrm{~g}$ of edible part, respectively. Those values were smaller than those found in Hangzhou-city (821, 


\section{7 and 543, respectively).}

The differences in n-3 PUFA content in three kinds of fish between two cities may be explained by the difference in the seasons of catch. The fish in Dalian-city were purchased in July, when Dalian was hot, and the fish in Hangzhou-city were purchased in December. It has long been known that fish contain more fat in winter. In fact sardines caught in Japan showed a wide range of fat content according to the season of catch. For instance, DHA content in the non-polar lipid fraction of sardine flesh varied from 2.4 weight $\%$ in August to $8.0 \%$ in December ${ }^{5)}$. There are a few Chinese epidemiological studies showing the healthy aspects of $n-3$ PUFA or fish eating. Men in Shanghai who consumed $\geq 200 \mathrm{~g}$ of fish/shellfish per week had a relative risk of 0.41 for fatal acute myocardial infarction (AMI) compared with men consuming $<50 \mathrm{~g}$ per week ${ }^{6}$. However, the algorithm used in their study for the assessment of $n-3$ PUFA intakes was rather simple. They assigned the following constants to three categories of fish: $0.57 \mathrm{~g} \mathrm{n}-3$ PUFA per $100 \mathrm{~g}$ fresh fish, $0.44 \mathrm{~g}$ per $100 \mathrm{~g}$ salted fish, and $0.36 \mathrm{~g}$ per $100 \mathrm{~g}$ shellfish ${ }^{6}$. $\mathrm{n}-3$ PUFA intakes were not calculated in many of the other epidemiological studies with Chinese subjects ${ }^{3,7,8)}$. Databases of the fatty acid composition of Chinese fish are necessary to better estimate the fatty acid composition of diet. Our data were limited in terms of the area and season of catch, and the number of species. However, our database would serve as the starting point for the fatty acid composition study of Chinese fish. Further studies are, therefore, necessary.

We are grateful to Ms Hiroko Hamatani and Ms Shizuko Takebe for their technical assistance. This work was partly supported by Polyene Project Corporation. 


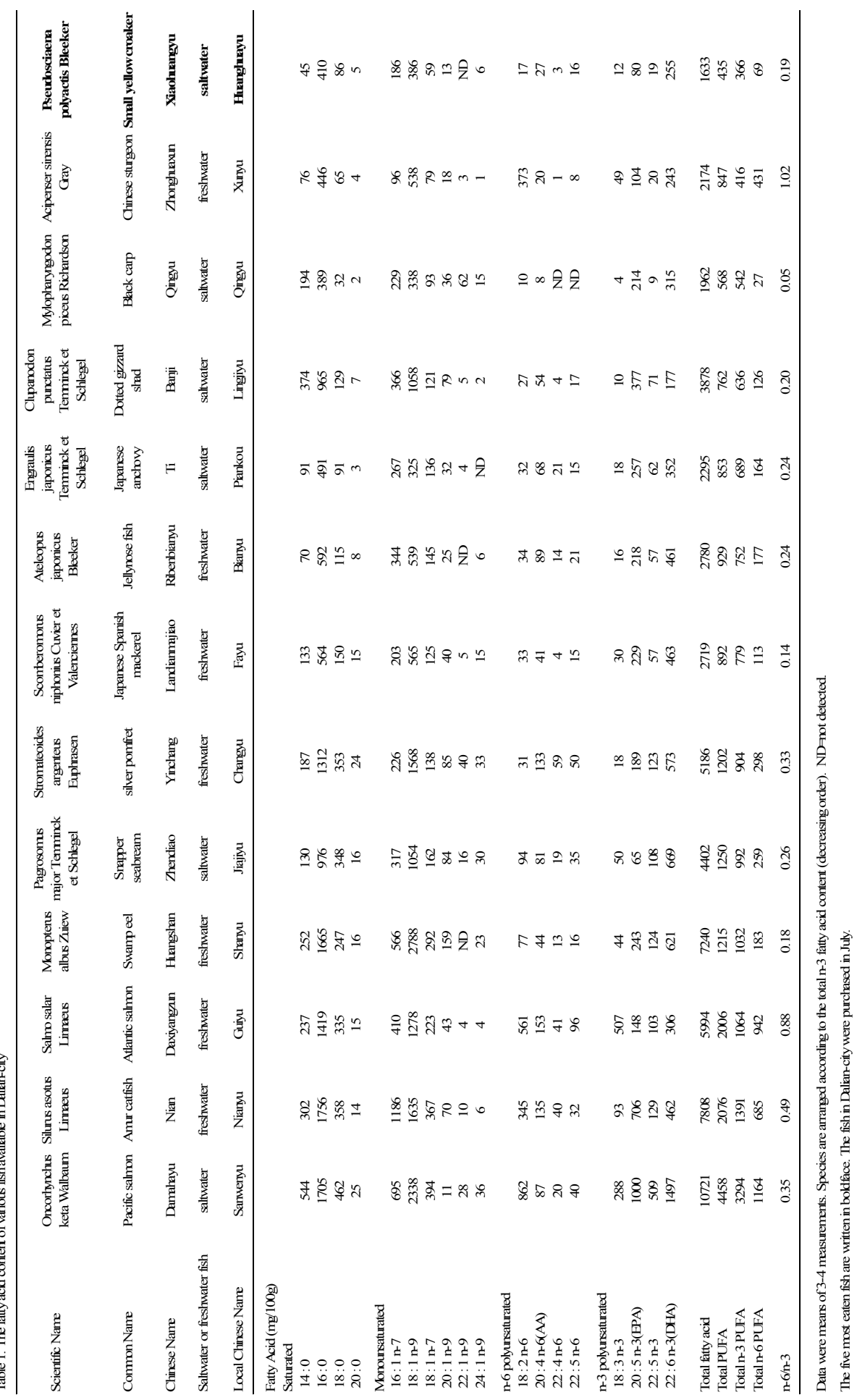




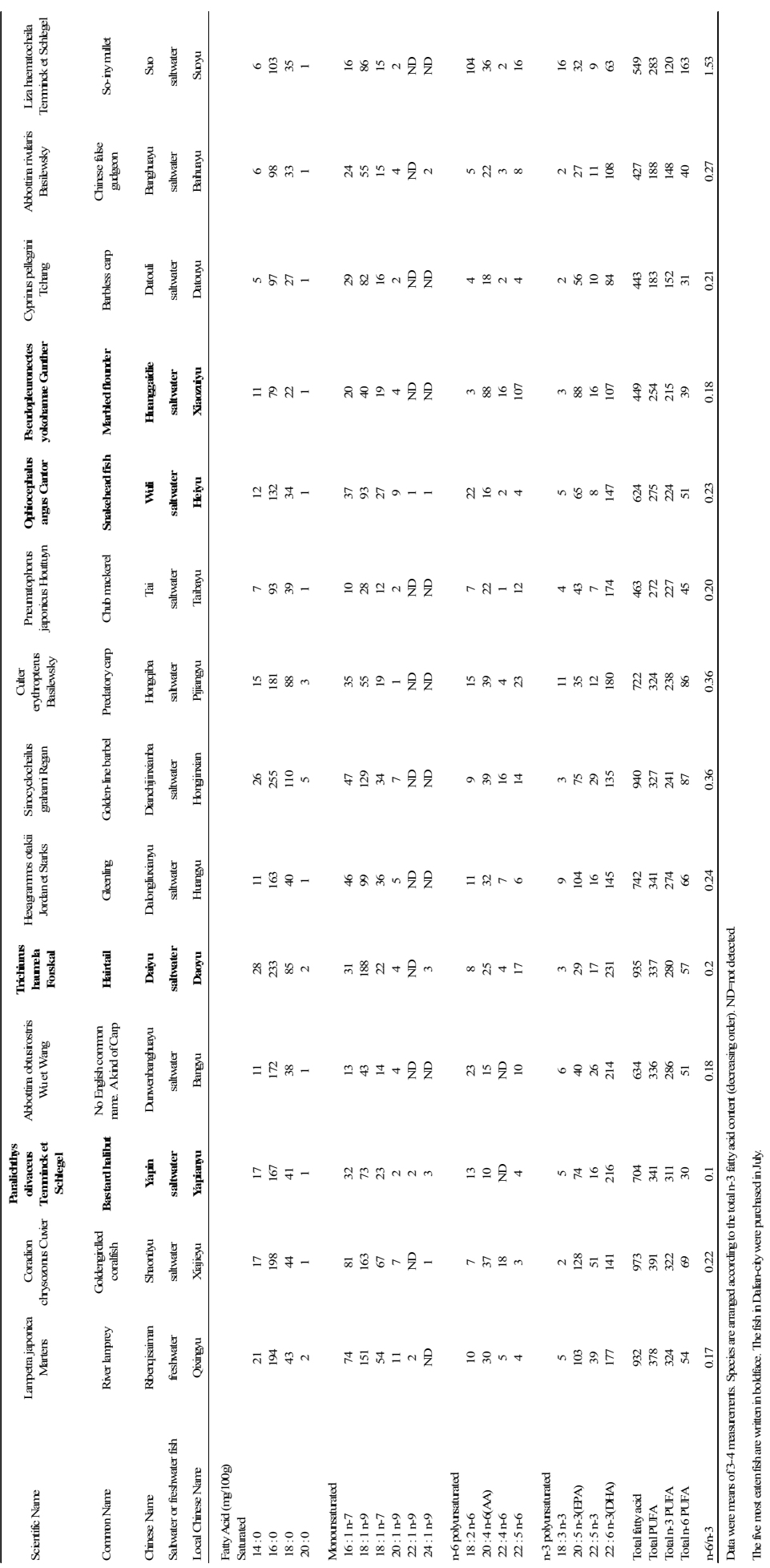




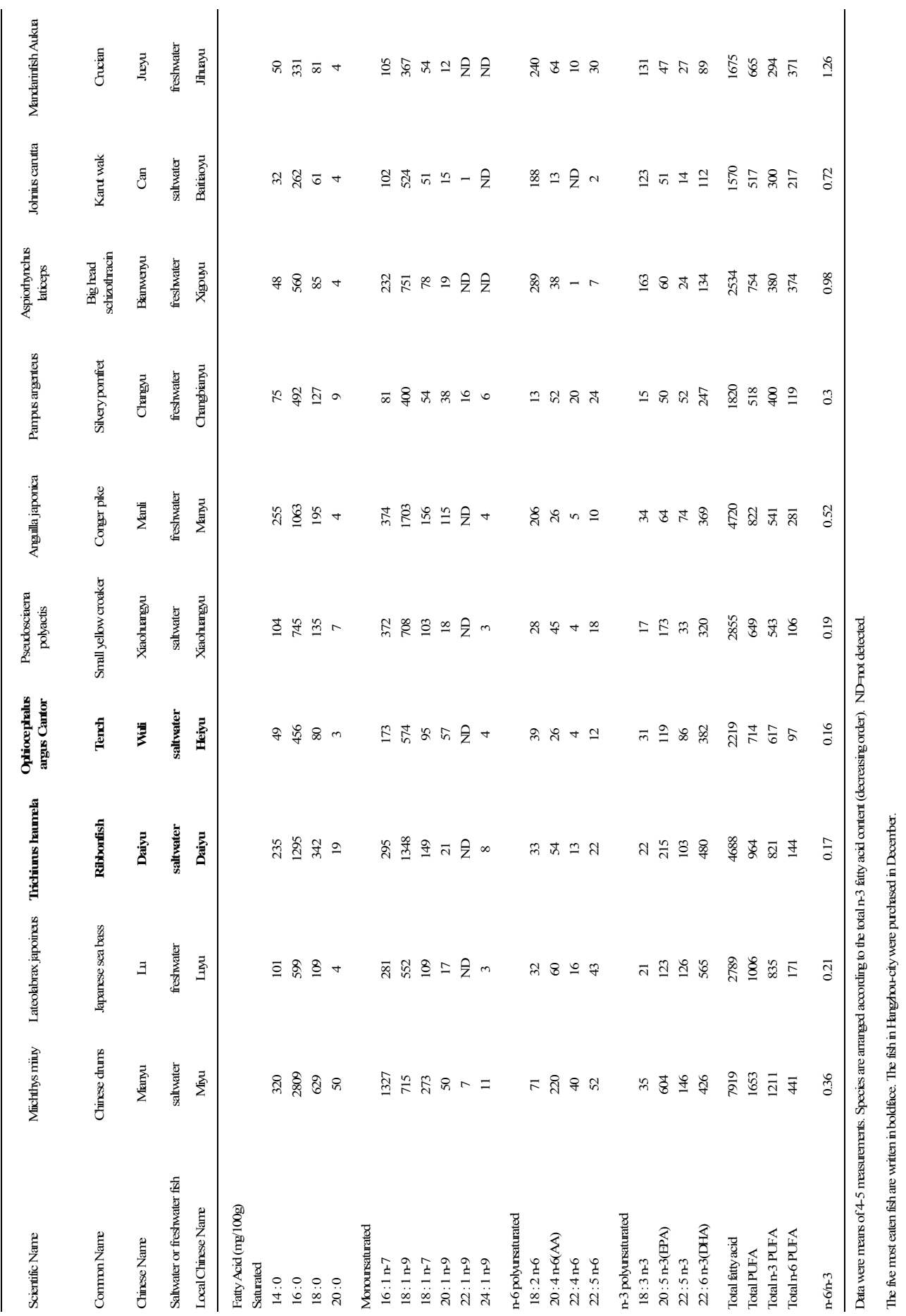




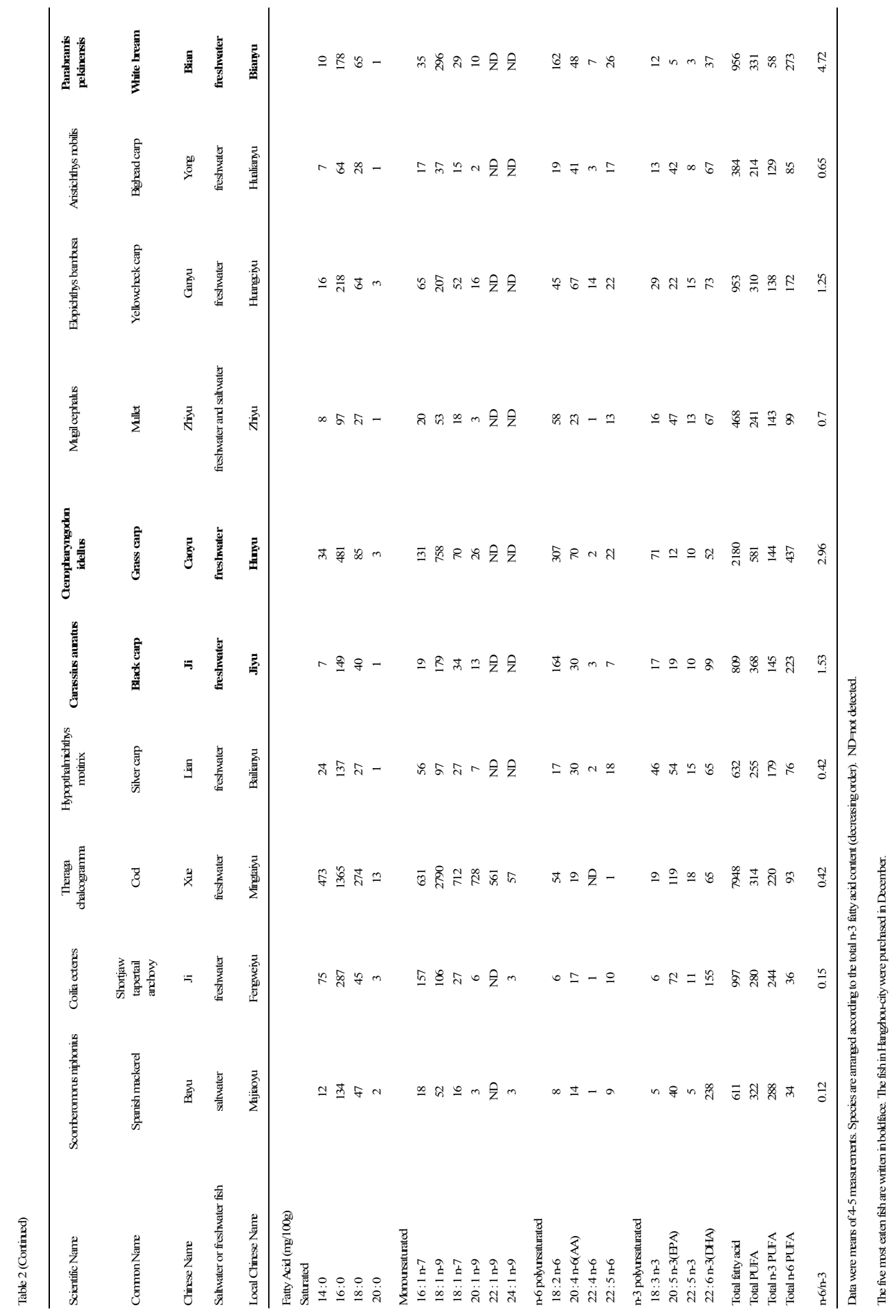




\section{REFERENCES}

1. Wang XC and Chen JQ. Guangzhou Food Sci Technol, 20: 81-82, 2004. (in Chinese)

2. Yang JG, Li GL, Jin QH, Chen DH and He SW. J Tongji Med Univ, 14: 77-80, 1994.

3. Huan M, Hamazaki K, Sun Y, Itomura M, Liu H, Kang W, Watanabe S, Terasawa K and Hamazaki T. Biol Psychiatry, 56: 490-496, 2004.

4. Japan Food Research Laboratories. Jitsumushano-kaita 5tei nihonshokuhin-hyojunseibun bunseki-manualno kaisetsu. (Japan Food Research Laboratories, Edit) pp 277-280, Tokyo. Chuohoki, Tokyo, 2001. (in Japanese)

5. Hayashi K and Takagi T. S Bull Fac Fish Hokkaido Univ, 28: 83-94, 1977.

6. Yuan J-M, Ross RK, Gao Y-T and Yu MC. Am J Epidemiol, 154: 809-816, 2001.

7. Hankin JH, Stram DO, Arakawa K, Park S, Low SH, Lee HP and Yu MC. Nutr Cancer, 39: 187-195, 2001.

8. Woo J, Leung SS, Ho SC, Sham A, Lam TH and Janus ED. Eur. J. Clin. Nutr, 53: 461-467, 1999. 